\title{
Massa específica da madeira, rendimento da laminação e qualidade das lâminas de Pinus patula Schltdl \& Cham
}

\author{
Everton Lorenzett Tavares`, Éverton Hillig, Ezaquel Bednarczuk, João Fábio Machado, Erick \\ Mustefaga
}

Departamento de Engenharia Florestal, Universidade Estadual do Centro-Oeste, Irati, PR, Brasil.

\begin{abstract}
RESUMO Este estudo teve como objetivos determinar a massa específica básica da madeira, o rendimento da laminação e a qualidade das lâminas obtidas de Pinus patula; comparar os valores dessas variáveis com as obtidas para a tradicionalmente utilizada espécie Pinus taeda; e avaliar os efeitos da classe de diâmetro da árvore e da posição da madeira ao longo do fuste nestas variáveis, em árvores de três diferentes classes de diâmetro e com idade de 12 anos. Foram utilizadas árvores de três classes de diâmetro à altura do peito (17 a 22,99; 23 a 32,99 e $\geq 33 \mathrm{~cm}$ ) plantadas no município de Bituruna-PR. Verificou-se que a madeira de Pinus patula apresentou menor massa específica básica e que as suas árvores apresentaram maior número de toras passíveis de laminação e maior volume de madeira laminável que as de Pinus taeda. O rendimento médio em laminação não apresentou diferença estatística entre as duas espécies e a qualidade das lâminas também foi semelhante.
\end{abstract}

Palavras-chave: Propriedades físicas; processamento; qualidade do processamento.

\section{Wood density, peeling yield and veneers quality of Pinus patula Schaltdl \& Cham}

\begin{abstract}
This study aims to evaluate the wood density, peeling yield and veneer quality made from Pinus patula trees; to compare these values for Pinus patula with refereed and traditionally Pinus taeda species. Also, evaluating the effects of tree diameter classes and $\log$ position on these variables. Twelve-years-old trees of three classes classified through its diameters at breast height (17 to 22.99, 23 to 32.99 and $\geq 33 \mathrm{~cm}$ ) and cultivated in Bituruna-PR were used. It was concluded that Pinus patula wood presented lower density, higher number of peelable logs and higher volume of produced veneers than Pinus taeda wood. The average peeling yield did not present statistical difference between the two species, and the veneers quality was similar. Keywords: Physical properties; processing; quality of qrocessing.
\end{abstract}

\section{Introdução}

No Brasil, a produção de lâminas e compensados movimenta uma grande fatia de mercado, suprida por mais 200 fábricas de compensado que detêm uma capacidade instalada de produção de mais de 4 milhões de $\mathrm{m}^{3}$ anuais. As regiões Sul e Sudeste produzem compensado de pinus, que supera a produção de compensado tropical em aproximadamente 200\% (VIEIRA et al., 2012).

O estado do Paraná concentra 81 empresas produtoras de lâminas e compensados, sendo a principal madeira utilizada do gênero Pinus (RIBASKI et al., 2016). Segundo Eisnfeld; Nascimento (2015), os plantios de pinus no Paraná somam 653.566 ha, que correspondem a $61 \%$ dos plantios florestais do estado. No entanto, os plantios da espécie Pinus taeda, que é plantada por diversas empresas florestais do estado, sofrem com ataques frequentes de pragas, sendo uma delas o macaco-prego (LIEBSCH, 2016).

Para obter-se uma economia sustentável e um melhor desenvolvimento dentro deste setor é importante que espécies 
alternativas sejam inseridas nas linhas de produção deste produto. Isto propicia uma distribuição da exploração de matéria-prima, tornando o processo de laminação mais versátil.

O Pinus patula Schltdl \& Cham é um excelente candidato para a produção de lâminas e trata-se de uma espécie originária do México, conhecida, entre outros nomes locais, como pinheiro de folhas penduradas. Suas árvores podem alcançar até $30 \mathrm{~m}$ de altura e diâmetro à altura do peito (DAP) de até 1,2 m, produzindo dessa forma grande volume de madeira. Sua madeira é usada para produção de polpa em diversas fábricas na África do Sul e foi considerada apropriada para produção de painéis aglomerados de boa resistência mecânica (ORWA et al., 2009).

De forma geral, as características demandadas da árvore para sua laminação são: massa específica de baixa a média amplitude (menor que 0,6 g. $\mathrm{cm}^{-3}$ ), fuste cilíndrico e retilíneo, grã direita ou levemente inclinada, estabilidade dimensional, e outras que definem a melhor trabalhabilidade da madeira (ABIMCI, 2004).

A massa específica da madeira é uma propriedade determinante para a obtenção de laminação de alta qualidade, pois ela está, mesmo que indiretamente, relacionada às respostas da madeira quando sobre estresse mecânico. Segundo Mattos et al. (2011), a massa específica pode ser útil para a avaliação da qualidade da madeira, sendo um indicativo das suas outras propriedades físicas e mecânicas e podendo servir de parâmetro para sua utilização.

Trabalhos recentemente publicados exemplificam a relação entre a massa específica e as propriedades mecânicas, e utilizam tais comparações como fator para expressar a qualidade da madeira. Juizo et al. (2015) avaliaram as propriedades físicas da madeira de Pinus patula com 38 anos de idade e obtiveram coeficiente de determinação de $73,46 \%$ para o modelo de estimativa da retratibilidade volumétrica em função da massa específica básica. Trianoski et al. (2014) verificaram forte relação entre a massa específica básica e as propriedades mecânicas da madeira de espécies de pinus tropicais, com coeficientes de correlação, na maioria dos casos, maiores que 0,70 .

Vários fatores influenciam a massa específica básica da madeira, dos quais se pode destacar: procedência da semente, espaçamento de plantio, taxa de crescimento, idade da árvore, posição da madeira no fuste, entre outros (VALÉRIO et al. 2008). Trevisan et al. (2008) destacam que a massa específica da madeira varia no sentido longitudinal e no sentido radial (medula/casca). Estas significativas variações ocorridas dentro do tronco estão em paralelo interligadas ao tamanho da árvore, o que torna importante a compreensão de sua variabilidade na obtenção de laminação de alto rendimento e qualidade.

Dessa forma, este estudo teve como objetivos determinar a massa específica básica da madeira, o rendimento da laminação e a qualidade das lâminas obtidas de Pinus patula; comparar os valores dessas variáveis com as obtidas para a espécie Pinus taeda; e avaliar os efeitos da classe de diâmetro da árvore e da posição da madeira ao longo do fuste nestas variáveis, em árvores de três diferentes classes de diâmetro e com idade de 12 anos.

\section{Material e Métodos}

\section{Área de estudo}

A região de estudo está localizada no município de Bituruna, região Centro-Sul do estado do Paraná. Com altitude média de 1.300 m, sendo classificado por Köppen, em clima temperado $(\mathrm{Cfb})$, com temperatura média no mês mais frio abaixo de $18^{\circ} \mathrm{C}$ (mesotérmico), com verões frescos, temperatura média no mês mais quente abaixo de $22^{\circ} \mathrm{C}$ e sem estação seca definida (IAPAR, 2015a).

A temperatura média da região está entre 22,6 e $12,0^{\circ} \mathrm{C}$, a precipitação e umidade relativa do ar média mensal são de, 
respectivamente, $176,25 \mathrm{~mm}$ e 77,2\% (IAPAR, 2015b). O plantio apresenta espaçamento 3,0 x 2,0 m e 12 anos de idade.

\section{Características dendrométricas das árvores de}

\section{Pinus patula e Pinus taeda}

Com os dados do inventário do plantio florestal estratificou-se três classes de diâmetro, deste modo, foram selecionadas seis árvores de cada classe de diâmetro de cada espécie, sendo usadas três árvores para determinação da densidade básica da madeira e três árvores para o processo de laminação. Foram coletados dados de diâmetro à altura do peito (DAP) e altura total da árvore (h). A partir destes dados foram calculadas as quantidades de toras passíveis de laminação (QTL) e o volume das árvores (v). O volume das árvores foi o comercial, obtido pelo método de Smalian, com a medição dos diâmetros na árvore em intervalos de $1 \mathrm{~m}$ e até o diâmetro mínimo de $5 \mathrm{~cm}$. A quantidade de toras lamináveis foi obtida seccionando as árvores em toras de 2,75 m de comprimento e considerando um diâmetro mínimo de $17 \mathrm{~cm}$ na extremidade de menor diâmetro da tora.

\section{Massa específica básica}

Para a determinação da massa específica básica optou-se pelo método utilizando discos, descrito na norma ABNT NBR 11941 (ABNT, 2003). A massa específica básica é a relação da massa seca em estufa a $105 \pm 2^{\circ} \mathrm{C}$ pelo respectivo volume da madeira saturada.

Foram retirados discos na base $(0,10 \mathrm{~m}), \mathrm{DAP}, 25,50 \mathrm{e}$ $70 \%$ da altura total das árvores. Os discos foram seccionados em quatro cunhas com ângulos de $90^{\circ}$, sendo todas utilizadas para a realização dos ensaios. As cunhas foram imersas em água durante 30 dias para garantir a saturação e, após esse período, foi determinado o volume por meio do método da balança hidrostática. Em seguida, as cunhas foram secas em estufa, primeiro à $50^{\circ} \mathrm{C}$ durante cinco dias e após a $105 \pm 2^{\circ} \mathrm{C}$ até massa constante, sendo então coletado o peso seco. Foram calculadas a massa específica básica média da árvore, pela média aritmética dos cinco discos coletados e pela média ponderada pelo volume de cada tora existente na seção entre os dis$\cos$.

\section{Rendimento da Laminação}

\section{Modelo experimental}

O modelo experimental consistiu num delineamento inteiramente casualizado (DIC) com seis tratamentos (Tabela 1). Das árvores, foram retiradas todas as toras passiveis de laminação, ou seja, as que apresentaram diâmetro mínimo de $17 \mathrm{~cm}$ na menor extremidade.

Tabela 1. Tratamentos empregados para os testes.

Table 1. Experimental design for the test.

\begin{tabular}{ccc}
\hline Tratamento & Espécie & Classe de diâmetro $(\mathbf{c m})$ \\
\hline T1 & Pinus patula & $17,00-22,99$ \\
T2 & Pinus taeda & \\
\hline T3 & Pinus patula & $23,00-32,99$ \\
T4 & Pinus taeda & \\
T5 & Pinus patula & $\geq 33,00$ \\
T6 & Pinus taeda & \\
\hline
\end{tabular}

\section{Produção das lâminas e rendimento da laminação}

No processo de laminação, as toras coletadas permaneceram durante $24 \mathrm{~h}$ na câmara de cozimento a vapor, com temperatura de $60^{\circ} \mathrm{C}$. As toras foram então torneadas com espessura nominal de 2,2 $\mathrm{mm}$ em torno com fuso de $8 \mathrm{~cm}$ de diâmetro. Para o cálculo de rendimento da laminação, procedeuse a cubagem das toras com casca antes da laminação, após a remoção da casca e o arredondamento, e após o final do processo de laminação (rolo-resto).

Após a formação do tapete de lâminas no torno, ocorreu o seccionamento das lâminas com guilhotina. As lâminas nas foram seccionadas nas dimensões de 0,9 x 1,35 m. Após o desenrolo e guilhotinagem, procedeu-se a contagem do número de lâminas e classificação de lâminas de cada tora. 
No processo de laminação, as perdas foram determinadas em três momentos, sendo: a) ocorridas no descascamento e arredondamento, b) ocorridas no rolo resto e c) perdas com o manuseio e lâminas segregadas. As perdas com descascamento e arredondamento correspondem basicamente ao efeito da conicidade e defeitos na forma da tora. Já as perdas relacionadas ao rolo resto são dependentes do tipo de equipamento utilizado e as demais perdas são relacionadas ao manuseio operacional das lâminas. Neste estudo, não foram consideradas as influências relacionadas às condições operacionais do equipamento.

O cálculo do rendimento efetivo ( $R$ ) foi realizado com base no volume do fardo e no volume da tora, sendo o fardo composto pelo total de lâminas inteiras obtidas de cada tora. Os demais cálculos foram efetuados por meio das diferenças entre cada etapa, resultando nas perdas de laminação, por classe de diâmetro.

Para classificação da qualidade das lâminas foram utilizadas as normas ABNT, NBR ISO 2426-1 e 2426-3 (ABNT, 2006a e ABNT, 2006b) e os parâmetros de qualidade definidos no catálogo técnico da ABIMCI (ABIMCI, 2002). Ambas definem parâmetros de qualidade do compensado por meio da qualidade das lâminas de face e contra face que, neste caso, foram utilizados para classificação de cada lâmina utilizada para produção dos painéis. Por ser a mais difundida, a denominação utilizada na classificação foi a do catálogo técnico da ABIMCI, que define as classes de qualidade em $\mathrm{A}, \mathrm{B}, \mathrm{C}+, \mathrm{C} \mathrm{e}$ D.

\section{Análise estatística}

A análise de massa específica básica foi realizada em três etapas. Primeiro foram calculados os valores de massa específica básica ponderada $\left(\mathrm{MEB}_{\mathrm{MP}}\right)$, da massa específica básica aritmética $\left(\mathrm{MEB}_{\mathrm{MA}}\right)$ e da massa específica básica nas diferentes alturas do tronco $\left(\mathrm{MEB}_{\text {Base }}, \mathrm{MEB}_{\mathrm{DAP}}, \mathrm{MEB}_{25 \%}, \mathrm{MEB}_{50 \%} \mathrm{e}\right.$ $\mathrm{MEB}_{70 \%}$ ) da madeira de ambas as espécies. Após, foi realizada a análise estatística em duas etapas, sendo a primeira a análise da $\mathrm{MEB}_{\mathrm{MP}}$ e da $\mathrm{MEB}_{\mathrm{MA}}$, no esquema fatorial, considerando os fatores espécie e classe de diâmetro. A segunda análise, também no esquema fatorial, considerou os fatores classe de diâmetro e posição ao longo do fuste para cada espécie.

Para o rendimento da laminação, os valores médios das características dendrométricas das árvores e os valores médios das variáveis de rendimento da laminação foram submetidos à análise de variância no esquema fatorial considerando os fatores espécie e classe de diâmetro.

Satisfeitas as prerrogativas de homogeneidade das variâncias pelo teste de Bartlett e distribuição normal dos resíduos pelo teste Kolmogorov-Smirnov, foi aplicada a análise de variância com $5 \%$ de probabilidade de erro, para os fatores e suas interações. Havendo diferenças significativas, foi aplicado o teste de Tukey para a comparação das médias.

\section{Resultados e Discussão}

Os valores médios encontrados para massa específica básica $(\mathrm{MEB})$ aritmética $\left(\mathrm{MEB}_{\mathrm{MA}}\right)$ e ponderada $\left(\mathrm{MEB}_{\mathrm{MP}}\right)$ das madeiras de Pinus patula e Pinus taeda, juntamente com os valores de MEB em cada posição no sentido longitudinal das árvores estão apresentados na Tabela 2.

Observa-se uma redução na massa específica básica com o aumento da altura no sentido longitudinal da árvore, efeito considerado normal e observado nas duas espécies. Embora as árvores tenham apenas 12 anos e provavelmente ainda sem madeira adulta, os anéis de crescimento são maiores nas partes mais altas do fuste, por ser uma parte mais jovem da árvore, e isso contribui para diminuir a massa específica da madeira. 
Tabela 2. Valores médios da massa específica básica ponderada $\left(\mathrm{MEB}_{\mathrm{MP}}\right)$, da massa específica básica aritmética e da massa específica básica nas diferentes alturas do tronco da madeira de Pinus patula e de Pinus taeda aos 12 anos.

Table 2. Average weighted density and arithmetic density values, as well as density measured over the trunk height obtained for 12-year-old Pinus paulta and Pinus taeda trees.

\begin{tabular}{|c|c|c|c|c|c|c|c|c|c|}
\hline \multirow{2}{*}{ TRAT. } & \multirow{2}{*}{ Espécie } & \multirow{2}{*}{$\begin{array}{c}\text { Classe Diâmetro } \\
(\mathrm{cm})\end{array}$} & \multirow{2}{*}{$\begin{array}{l}\mathrm{MEB}_{\mathrm{MP}} \\
\left(\mathrm{g} . \mathrm{cm}^{-3}\right)\end{array}$} & \multirow{2}{*}{$\begin{array}{l}\operatorname{MEB}_{\mathrm{MA}} \\
\left(\mathrm{g} . \mathrm{cm}^{-3}\right)\end{array}$} & \multicolumn{5}{|c|}{ Posições ao longo do fuste $\left(\mathrm{g} \cdot \mathrm{cm}^{-3}\right)$} \\
\hline & & & & & MEB $_{\text {Base }}$ & MEB $_{\text {DAP }}$ & MEB $_{25 \%}$ & MEB $_{50 \%}$ & MEB $_{70 \%}$ \\
\hline \multirow[t]{2}{*}{ T1 } & P. patula & $17,00-22,99$ & 0,34 & 0,34 & 0,37 & 0,35 & 0,34 & 0,33 & 0,32 \\
\hline & & & $(0,019)$ & $(0,025)$ & $(0,003)$ & $(0,010)$ & $(0,025)$ & $(0,014)$ & $(0,029)$ \\
\hline \multirow[t]{2}{*}{$\mathrm{T} 2$} & P. taeda & $17,00-22,99$ & 0,36 & 0,36 & 0,40 & 0,39 & 0,36 & 0,34 & 0,33 \\
\hline & & & $(0,010)$ & $(0,031)$ & $(0,010)$ & $(0,008)$ & $(0,011)$ & $(0,012)$ & $(0,029)$ \\
\hline \multirow[t]{2}{*}{ T3 } & P. patula & $23,00-32,99$ & 0,31 & 0,33 & 0,37 & 0,36 & 0,31 & 0,30 & 0,29 \\
\hline & & & $(0,009)$ & $(0,039)$ & $(0,027)$ & $(0,035)$ & $(0,002)$ & $(0,015)$ & $(0,013)$ \\
\hline \multirow[t]{2}{*}{$\mathrm{T} 4$} & P. taeda & $23,00-32,99$ & 0,36 & 0,37 & 0,42 & 0,39 & 0,37 & 0,35 & 0,33 \\
\hline & & & $(0,006)$ & $(0,037)$ & $(0,011)$ & $(0,023)$ & $(0,018)$ & $(0,007)$ & $(0,032)$ \\
\hline \multirow[t]{2}{*}{ T5 } & P. patula & $\geq 33,00$ & 0,31 & 0,31 & 0,33 & 0,31 & 0,31 & 0,30 & 0,30 \\
\hline & & & $(0,016)$ & $(0,017)$ & $(0,018)$ & $(0,014)$ & $(0,016)$ & $(0,017)$ & $(0,016)$ \\
\hline \multirow[t]{6}{*}{$\mathrm{T} 6$} & P. taeda & $\geq 33,00$ & 0,33 & 0,34 & 0,38 & 0,37 & 0,34 & 0,31 & 0,30 \\
\hline & & & $(0,010)$ & $(0,036)$ & $(0,028)$ & $(0,022)$ & $(0,016)$ & $(0,007)$ & $(0,007)$ \\
\hline & P. patula & & 0,32 & 0,33 & 0,36 & 0,34 & 0,32 & 0,31 & 0,30 \\
\hline & & Média & $(0,020)$ & $(0,031)$ & $(0,032)$ & $(0,031)$ & $(0,030)$ & $(0,030)$ & $(0,030)$ \\
\hline & P. taeda & & 0,35 & 0,36 & 0,40 & 0,39 & 0,35 & 0,33 & 0,32 \\
\hline & & & $(0,016)$ & $(0,036)$ & $(0,033)$ & $(0,032)$ & $(0,032)$ & $(0,035)$ & $(0,038)$ \\
\hline
\end{tabular}

Mattos et al. (2011) também observaram diminuição da massa específica básica da madeira com aumento da altura no fuste para Pinus taeda de 13 anos de idade.

Na Tabela 3 é apresentada a análise de variância fatorial da massa específica básica média da árvore (aritmética e ponderada pelo volume) em função dos fatores espécie e classe de diâmetro da árvore. Verifica-se que a massa específica básica de ambas as espécies sofreu efeito da taxa de crescimento, pois a maior classe de diâmetro apresentou menor massa específica básica que as classes inferiores. A maior classe de diâmetro apresentou anéis de crescimento maiores em ambas as espécies e isso foi o fator que determinou a menor massa específica básica de sua madeira.

Rezende et al. (2007) verificaram uma variação diferente para Pinus tecunumannii de 14 anos, que apresentou madeira de menor massa específica básica na classe de diâmetro intermediária $\left(0,422 \mathrm{~g} \cdot \mathrm{cm}^{-3}\right.$ para $\left.26-30 \mathrm{~cm}\right)$ em relação às classes inferior $\left(0,440 \mathrm{~g} . \mathrm{cm}^{-3}\right.$ para $\left.20-25 \mathrm{~cm}\right)$ e superior $(0,436 \mathrm{~g} . \mathrm{cm}-$
${ }^{3}$ para $31-37 \mathrm{~cm}$ ), embora sem citar o tamanho dos anéis de crescimento.

Tabela 3. Análise fatorial para massa específica básica aritmética e massa específica básica ponderada, considerando os fatores espécie e classe de diâmetro.

Table 3. Factorial analysis of the average weighted density, and arithmetic density as a function of specie and diameter class.

\begin{tabular}{cccc}
\hline Fator & & MEB $_{\mathrm{MA}}$ & MEB $_{\mathrm{MP}}$ \\
\hline \multirow{2}{*}{ Espécie } & $\begin{array}{c}\text { P. patula } \\
\text { P. taeda }\end{array}$ & $0,33 \mathrm{~b}$ & $0,32 \mathrm{~b}$ \\
& & $28,596^{*}$ & $29,535^{\star}$ \\
\hline$F_{\text {calc }}$ & $17,00-22,99$ & $0,35 \mathrm{a}$ & $0,34 \mathrm{a}$ \\
Classe de Diâmetro & $23,00-32,99$ & $0,35 \mathrm{a}$ & $0,34 \mathrm{a}$ \\
$(\mathrm{CD})$ & $\geq 33,00$ & $0,32 \mathrm{~b}$ & $0,32 \mathrm{~b}$ \\
\hline$F_{\text {calc }}$ & & $8,630^{\star}$ & $8,141^{\star}$ \\
\hline Interação $F_{\text {calc }}$ & & $1,625 \mathrm{~ns}$ & $2,664 \mathrm{~ns}$ \\
\hline
\end{tabular}

As médias seguidas pela mesma letra não diferem estatisticamente entre si. Foi aplicado o Teste de Tukey; ${ }^{*}$ : significativo a $5 \%$ de probabilidade de erro; ns: não significativo.

Na Tabela 4 são apresentadas as médias e a análise estatística para a $\mathrm{MEB}_{\mathrm{MA}}$ de ambas as espécies, considerando os fatores classe de diâmetro e posição ao longo do fuste. 
Tabela 4. Análise fatorial para massa específica básica calculada pela média aritmética $\left(\mathrm{MEB}_{\mathrm{MA}}\right)$ de cada espécie, considerando diâmetro e posição ao longo do fuste.

Table 4. Factorial analysis of the arithmetic density (MEB $\left.\mathrm{MA}_{\mathrm{MA}}\right)$ of each species, as a function of diameter class and trunk position.

\begin{tabular}{|c|c|c|c|}
\hline \multirow{2}{*}{ Fator } & & \multicolumn{2}{|c|}{$\operatorname{MEB}_{\mathrm{MA}}\left(\mathrm{g} \cdot \mathrm{cm}^{-3}\right)$} \\
\hline & & P. taeda & P. patula \\
\hline Classe de & $17,00-22,99$ & $0,36 \mathrm{a}$ & $0,34 \mathrm{a}$ \\
\hline Diâmetro & $23,00-32,99$ & $0,37 \mathrm{a}$ & $0,32 \mathrm{~b}$ \\
\hline$(\mathrm{CD})$ & $\geq 33,00$ & $0,34 \mathrm{~b}$ & $0,31 \mathrm{c}$ \\
\hline$F_{\text {calc }}$ & & $10,746^{*}$ & $12,822^{\star}$ \\
\hline \multirow{5}{*}{$\begin{array}{c}\text { Posição ao } \\
\text { longo do fuste }\end{array}$} & $0,1 \mathrm{~m}$ & $0,40 \mathrm{a}$ & $0,35 \mathrm{a}$ \\
\hline & $1,3 \mathrm{~m}$ & $0,39 \mathrm{a}$ & $0,34 \mathrm{ab}$ \\
\hline & $25 \% \mathrm{~h}$ & $0,35 \mathrm{~b}$ & $0,32 \mathrm{bc}$ \\
\hline & $50 \% \mathrm{~h}$ & $0,33 \mathrm{bc}$ & $0,31 \mathrm{c}$ \\
\hline & $70 \% \mathrm{~h}$ & $0,32 \mathrm{c}$ & $0,30 \mathrm{c}$ \\
\hline$F_{\text {calc }}$ & & $29,289^{*}$ & $12,546^{*}$ \\
\hline Interação $F_{\text {calc }}$ & & $0,707 \mathrm{~ns}$ & $1,832 \mathrm{~ns}$ \\
\hline
\end{tabular}

As médias seguidas pela mesma letra não diferem estatisticamente entre si. Foi aplicado o Teste de Tukey; ${ }^{*}$ : significativo a $5 \%$ de probabilidade de erro; ns: não significativo.

A massa específica básica $\left(\mathrm{MEB}_{\mathrm{MA}}\right)$ diminui ao longo do fuste da árvore para ambas as espécies, mas observa-se ainda que a variação da $\mathrm{MEB}_{\mathrm{MA}}$ do Pinus patula $\left(0,05 \mathrm{~g} . \mathrm{cm}^{-3}\right)$ é menor que a do Pinus taeda $\left(0,08\right.$ g. $\left.\mathrm{cm}^{-3}\right)$, indicando menor variação para as árvores dessa espécie. Na Figura 1 é apresentado o gráfico das médias e da dispersão dos dados de MEB para cada espécie e para cada posição no fuste. Observa-se uma variação significativa de $\mathrm{MEB}$ na mesma posição do fuste que, em parte, pode ser explicada pela variação de classes de diâmetro das árvores.

Verificou-se que toras obtidas até $25 \%$ da altura total na árvore são indicadas para laminação, por apresentarem diâmetros médios compatíveis com os tornos laminadores. Em geral, as toras apropriadas para os tornos laminadores devem apresentar diâmetros entre 20 e $40 \mathrm{~cm}$, sendo que neste estudo foi considerado o diâmetro mínimo de $17 \mathrm{~cm}$.

Pode-se observar que houve diferença entre as classes de diâmetro para todas variáveis dendrométricas avaliadas e para as espécies houve diferença estatística na altura total (h), na quantidade de toras $\left(\mathrm{Q}_{\mathrm{T}}\right)$ e no volume em tora laminável $\left(\mathrm{V}_{\mathrm{TL}}\right)$ (Tabela 5).

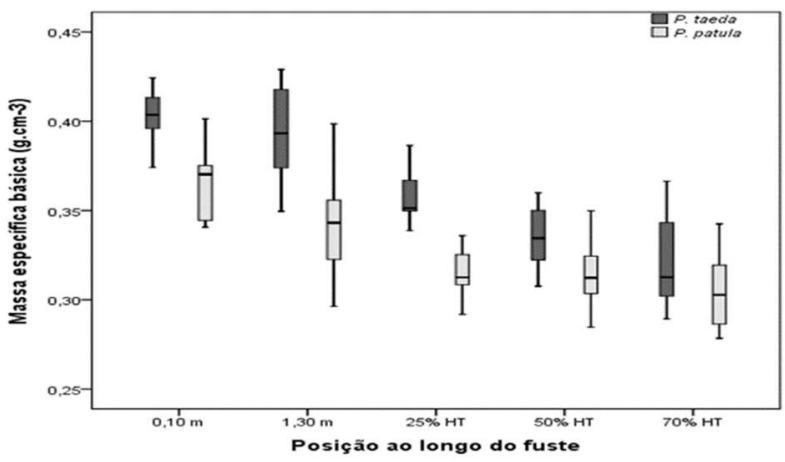

Figura 1. Boxplot da massa específica básica por posição ao longo do fuste para cada espécie estudada.

Figure 1. Boxplot of density as a function of trunk position for each species.

As árvores de Pinus patula apresentaram valor médio de volume em tora laminável $36 \%$ maior que as de Pinus taeda na maior classe de diâmetro, demonstrando bom potencial dessa espécie para laminação. Esse fato está relacionado ao maior crescimento das árvores de Pinus patula, pois mesmo não havendo diferença estatística entre o DAP das duas espécies, a altura total e a quantidade de toras foram maiores para essa espécie.

Na Tabela 6 são apresentados os valores médios de rendimento e de perdas no processo de laminação por classe de diâmetro e por espécie. Verificou-se 43,22 e 40,16\% de rendimento médio para Pinus patula e para Pinus taeda, respectivamente, valores inferiores aos resultados obtidos em pesquisas com espécies do gênero como Bortoletto Júnior (2008) de 57,4\% para Pinus merkusii de 26 anos e Almeida et al. (2014) de 52,7\% para Pinus elliottii de 7 anos e 10 meses. 
Tabela 5. Valores médios das características dendrométricas das árvores de Pinus patula e de Pinus taeda.

Table 5. Average values for the dendrometric characteristics of the Pinus patula and Pinus taeda trees.

\begin{tabular}{ccccccccc}
\hline TRAT. & Espécie & $\begin{array}{c}\text { C. } \mathbf{D} . \\
(\mathbf{c m})\end{array}$ & DAP $(\mathbf{c m})$ & $\begin{array}{c}\mathbf{h} \\
(\mathbf{m})\end{array}$ & $\begin{array}{c}\mathbf{Q}_{\mathrm{T}} \\
(\mathbf{u n d})\end{array}$ & $\begin{array}{c}\mathbf{v} \\
\left(\mathbf{m}^{\mathbf{3}}\right)\end{array}$ & $\begin{array}{c}\mathbf{Q}_{\mathrm{TL}} \\
(\mathbf{u n d})\end{array}$ & $\begin{array}{c}\mathbf{V}_{\mathrm{TL}} \\
\left(\mathbf{m}^{\mathbf{3}}\right)\end{array}$ \\
\hline T1 & P.patula & $17,00-22,99$ & $21,63 \mathrm{c}$ & $19,85 \mathrm{~b}$ & $4 \mathrm{c}$ & $0,364 \mathrm{~cd}$ & $2 \mathrm{bc}$ & $0,295 \mathrm{~cd}$ \\
T2 & P. taeda & $17,00-22,99$ & $20,17 \mathrm{c}$ & $16,17 \mathrm{c}$ & $4 \mathrm{c}$ & $0,257 \mathrm{~d}$ & $1 \mathrm{c}$ & $0,203 \mathrm{~d}$ \\
T3 & P.patula & $23,00-32,99$ & $25,77 \mathrm{~b}$ & $20,97 \mathrm{~b}$ & $5 \mathrm{ab}$ & $0,541 \mathrm{bc}$ & $4 \mathrm{ab}$ & $0,473 \mathrm{c}$ \\
T4 & P. taeda & $23,00-32,99$ & $26,68 \mathrm{~b}$ & $18,80 \mathrm{~b}$ & $4 \mathrm{bc}$ & $0,495 \mathrm{~cd}$ & $3 \mathrm{bc}$ & $0,363 \mathrm{~cd}$ \\
T5 & P.patula & $\geq 33,00$ & $35,38 \mathrm{a}$ & $23,67 \mathrm{a}$ & $6 \mathrm{a}$ & $1,062 \mathrm{a}$ & $5 \mathrm{a}$ & $0,984 \mathrm{a}$ \\
T6 & P. taeda & $\geq 33,00$ & $34,00 \mathrm{a}$ & $18,50 \mathrm{bc}$ & $4 \mathrm{bc}$ & $0,798 \mathrm{ab}$ & $4 \mathrm{ab}$ & $0,723 \mathrm{~b}$ \\
\hline & P.patula & \multirow{2}{*}{ Média } & 27,59 & 21,50 & 5 & 0,656 & 4 & 0,584 \\
& & 26,95 & 17,82 & 4 & 0,517 & 3 & 0,430 \\
\hline
\end{tabular}

TRAT: Tratamento / C.D.: Classe de diâmetro / DAP: Diâmetro a altura do peito / h: Altura total da árvore / QT: Quantidade toras / v: Volume da árvore / QTL: Quantidade de toras para laminação / $\mathrm{V}_{\text {TL: }}$ Volume em tora laminável. As médias seguidas pela mesma letra não diferem estatisticamente entre si. Foi aplicado o Teste de Tukey a $5 \%$ de probabilidade erro

Tabela 6. Valores médios de rendimento e de perdas no processo de laminação das árvores de Pinus patula e de Pinus taeda. Table 6. Average values for the yield and losses during the veneering process of the Pinus patula and Pinus taeda trees.

\begin{tabular}{|c|c|c|c|c|c|c|c|}
\hline TRAT. & Espécie & $\begin{array}{l}\text { C.D. } \\
\text { (cm) }\end{array}$ & $\begin{array}{c}V_{F} \\
\left(m^{3}\right)\end{array}$ & $\begin{array}{c}\mathbf{R}_{\mathrm{TF}} \\
(\%)\end{array}$ & $\begin{array}{l}V_{R} \\
(\%)\end{array}$ & $\begin{array}{l}P_{1} \\
(\%)\end{array}$ & $\begin{array}{l}P_{2} \\
(\%)\end{array}$ \\
\hline $\mathrm{T} 1$ & P. patula & $17,00-22,99$ & $0,125 \mathrm{c}$ & $39,63 \mathrm{a}$ & $18,59 \mathrm{ab}$ & $34,76 \mathrm{a}$ & $4,55 \mathrm{a}$ \\
\hline $\mathrm{T} 2$ & P. taeda & $17,00-22,99$ & $0,075 \mathrm{c}$ & $34,18 \mathrm{a}$ & $22,49 \mathrm{a}$ & $34,67 \mathrm{a}$ & $5,77 \mathrm{a}$ \\
\hline T3 & P. patula & $23,00-32,99$ & $0,219 \mathrm{bc}$ & $44,10 \mathrm{a}$ & $15,57 \mathrm{~b}$ & $28,50 \mathrm{a}$ & $9,23 \mathrm{a}$ \\
\hline $\mathrm{T} 4$ & P. taeda & $23,00-32,99$ & $0,192 \mathrm{bc}$ & $40,96 \mathrm{a}$ & $16,17 \mathrm{~b}$ & $30,80 \mathrm{a}$ & $18,34 \mathrm{a}$ \\
\hline T5 & P. patula & $\geq 33,00$ & $0,486 \mathrm{a}$ & $45,93 \mathrm{a}$ & $8,78 \mathrm{c}$ & $32,67 \mathrm{a}$ & $9,55 \mathrm{a}$ \\
\hline T6 & P. taeda & $\geq 33,00$ & $0,347 \mathrm{ab}$ & $45,35 \mathrm{a}$ & $8,15 \mathrm{c}$ & $26,09 \mathrm{a}$ & $18,00 \mathrm{a}$ \\
\hline & P. patula & \multirow{2}{*}{ Média } & 0,277 & 43,22 & 14,31 & 31,98 & 7,78 \\
\hline & P. taeda & & 0,205 & 40,16 & 15,60 & 30,52 & 14,04 \\
\hline
\end{tabular}

TRAT: Tratamento / C.D.: Classe de diâmetro / VF: Volume do Fardo de lâminas / RTF: Rendimento (Tora/Fardo) / $\mathrm{V}_{\mathrm{R}}=$ volume do rolo resto / $\mathrm{P}_{1}=$ perda com descascamento e arredondamento $/ \mathrm{P}_{2}=$ perdas com o manuseio e lâminas segregadas FFN: Fator de Forma Normal. As médias seguidas pela mesma letra não diferem estatisticamente entre si. Foi aplicado o Teste de Tukey a 95\% de probabilidade erro.

O menor rendimento obtido em relação às duas pesquisas foi decorrente do diâmetro das toras, pois embora a diferença de idade, nas duas pesquisas as árvores tinham DAP médio de $30 \mathrm{~cm}$ ou mais. No trabalho de Bortoletto Júnior (2008) foram laminadas 2 toras de 2,5 m cada a partir da base de cada árvore e no trabalho de Almeida et al. (2014) uma tora de 2,5 m a partir da base de cada árvore. Neste estudo, foram laminadas todas as toras até um diâmetro mínimo de $17 \mathrm{~cm}$ o que ocasionou um diâmetro médio de toras menor e, consequentemente, um menor rendimento na laminação. Dessa forma, verifica-se que o aproveitamento da árvore para múltiplos produtos e um maior diâmetro mínimo laminável do que 17 cm devem ser considerados na escolha das toras para laminação.

A média de perda por rolo resto foi de 14,3 e 15,6\% para Pinus patula e Pinus taeda, respectivamente. Essas perdas são influenciadas principalmente pelo diâmetro da tora e pelo equipamento, sendo semelhantes aos resultados encontrados por Almeida et al. (2014), de 15,11\% de perda com rolo-resto para Pinus elliottii.

A perda com o descascamento e arredondamento não diferiu estatisticamente entre as classes de diâmetro, e atribuiuse essas perdas à conicidade das árvores. Os resultados obtidos condizem com os da literatura quando se leva em consideração a idade das árvores, pois Almeida et al. (2014) obteve 
aproximadamente $56 \%$ de perda com descascamento e arredondamento na laminação de Pinus elliotti de 7 anos de idade.

Na Tabela 7 são apresentados os percentuais de número de lâminas obtidas com a classificação realizada conforme critérios definidos para ambas as espécies com base nas normas NBR ISO 2426-1 (ABNT, 2006a), NBR ISO 2426-3 (ABNT, 2006b) e ABIMCI (2002), por espécie e classe de diâmetro.

Em geral, as lâminas classificadas como A e B são utilizadas nas capas do painel compensado e as demais no miolo. Dessa forma, observa-se que as espécies apresentaram percentuais similares de lâminas de capa e de miolo. Em relação às classes de diâmetro observa-se, para ambas espécies, maiores porcentagens de lâminas classificadas como " $A$ " e " $B$ ” na maior classe.

Na Figura 2 é possível observar uma lâmina típica representativa de cada classe de qualidade para Pinus patula e para Pinus taeda. Uma lâmina classe A apresenta-se lisa e sem defeitos aparentes como nós, rachaduras, fendas de laminação, ondulações e bolsões de resina. A lâmina classificada como B pode apresentar pequenos defeitos, mas que se caracterizam mais como estéticos do que os que comprometem a sua resistência. As lâminas classificadas nas classes C+, C e D são as que apresentam um ou mais dos defeitos citados e sua classificação vai depender dos tipos e das quantidades de defeitos encontrados.

De modo geral, se verifica que a madeira de Pinus patula apresenta menor tamanho de nós, mas maior quantidade de bolsões e veios de resina do que a madeira de Pinus taeda. Em função disso, as lâminas de Pinus patula da classe D, embora tenham ocorrido em menor percentual, se apresentaram com maior proporção de defeitos que as de Pinus taeda.

A madeira de Pinus patula apresentou bolsões de resina localizados próximo à casca. Esse fator contribuiu para menor quantidade de lâminas classificadas como tipos "A" ou "B", do que as de Pinus taeda. Por outro lado, a madeira de Pinus patula apresentou menor quantidade de rachaduras e fendas nas lâminas o que contribuiu para menos lâminas na classe “D”. Esse fato, em parte, pode ser explicado pela massa específica da madeira, pois quanto menor o seu valor mais fácil aquecer o centro da tora e plasticizar a lignina.

Tabela 7. Percentuais de classe de qualidade das lâminas de Pinus patula e de Pinus taeda, por classe de diâmetro com base nas normas NBR ISO 2426-1; 2426-3 e ABIMCI (2002).

Table 7. Quality class percent of the veneers obtained from Pinus patula and Pinus taeda, as a function of class diameter based on the standards NBR ISO 2426-1; 2426-3 and ABIMCI (2002).

\begin{tabular}{|c|c|c|c|c|c|c|c|}
\hline \multirow{2}{*}{ Trat. } & \multirow{2}{*}{ Espécie } & \multirow{2}{*}{ C.D. $(\mathrm{cm})$} & \multicolumn{5}{|c|}{ Classificação das lâminas (\%) } \\
\hline & & & A & B & $\mathrm{C}+$ & $\mathrm{C}$ & D \\
\hline $\mathrm{T} 1$ & P. patula & $17,00-22,99$ & 0,0 & 13,3 & 35,7 & 51,0 & 0,0 \\
\hline $\mathrm{T} 2$ & P. taeda & $17,00-22,99$ & 9,8 & 0,0 & 46,3 & 31,7 & 12,2 \\
\hline T3 & P. patula & $23,00-32,99$ & 2,9 & 12,1 & 31,4 & 45,4 & 8,2 \\
\hline $\mathrm{T} 4$ & P. taeda & $23,00-32,99$ & 6,7 & 11,7 & 28,9 & 38,3 & 14,4 \\
\hline T5 & P. patula & $\geq 33$ & 7,9 & 18,9 & 40,9 & 22,2 & 10,0 \\
\hline T6 & P. taeda & $\geq 33$ & 11,2 & 20,8 & 33,7 & 19,4 & 14,8 \\
\hline & P. patula & & 3,6 & 14,8 & 36,0 & 39,5 & 6,1 \\
\hline & P. taeda & Dictia & 9,2 & 10,8 & 36,3 & 29,8 & 13,8 \\
\hline
\end{tabular}

Trat: Tratamento / C.D.: Classe de diâmetro. 

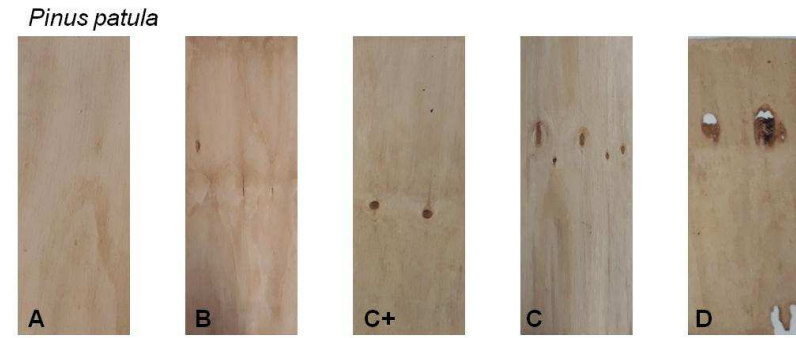

Pinus taeda
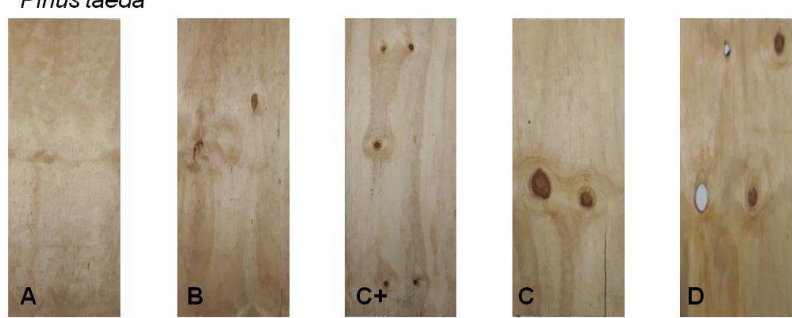

Figura 2. Lâminas típicas de cada classe de qualidade segundo critérios para ambas espécies com base nas normas NBR ISO 2426-1; 2426-3 e ABIMCI (2002).

Figure 2. Typical veneers obtained in each quality class as determined by NBR ISO 2426-1; 2426-3 and ABIMCI (2002).

\section{Conclusões}

A madeira de Pinus patula apresentou menor massa específica básica, calculadas para árvore pela média aritmética e pela média ponderada pelo volume, que a de Pinus taeda e a massa específica básica decresceu com a altura do fuste das árvores para ambas as espécies.

A classe de diâmetro influenciou na massa específica básica da madeira de Pinus patula, sendo diferente entre as três classes estudadas, e de Pinus taeda em que a classe de diâmetro acima de $33 \mathrm{~cm}$ apresentou madeira com menor massa específica básica que as outras classes.

As árvores de Pinus patula apresentaram maior volume de madeira laminável na maior classe de diâmetro, o rendimento médio tora/fardo e a qualidade das lâminas não apresentaram diferença estatística entre as duas espécies, confirmando o potencial de substituição da madeira de Pinus taeda pela de Pinus patula para laminação.

\section{Agradecimentos}

Os autores agradecem a CAPES - Coordenação de Aperfeiçoamento de Pessoal de Nível Superior, a UNICENTRO Universidade Estadual do Centro-Oeste e a REMASA Reflorestadora S/A pelo suporte recebido para desenvolvimento deste estudo.

\section{Referências}

ABIMICI - ASSOCIAÇÃO BRASILEIRA DA INDÚSTRIA DE MADEIRA PROCESSADA MECANICAMENTE. Compensado de Pinus - Catálogo Técnico n. 1, Curitiba, 2002. $24 \mathrm{p}$.

ABIMICI - ASSOCIAÇÃO BRASILEIRA DA INDÚSTRIA DE MADEIRA PROCESSADA MECANICAMENTE. Secagem de serrados e lâminas de madeira. Artigo Técnico No 27, Curitiba, 2004. 7p.

ABNT - ASSOCIAÇÃO BRASILEIRA DE NORMAS TÉCNICAS. Madeira - determinação da densidade básica. Rio de Janeiro, 2003. (Norma Brasileira NBR 11941).

ABNT - ASSOCIAÇÃO BRASILEIRA DE NORMAS TÉCNICAS. Madeira compensada - classificação pela aparência superficial, Parte 1: Geral. Rio de Janeiro, 2006a. (Norma Brasileira NBR ISO 2426-1).

ABNT - ASSOCIAÇÃO BRASILEIRA DE NORMAS TÉCNICAS. Madeira compensada - classificação pela aparência superficial, Parte 3: Coníferas. Rio de Janeiro, 2006b. (Norma Brasileira NBR ISO 2426-3).

ALMEIDA, N. F., BORTOLETTO JÚNIOR, G., MENDES, R. F., SURDI, P. G. Produção e avaliação da qualidade de lâminas de madeira de um híbrido de Pinus elliottii var. elliottii $\times$ Pinus caribaea var. hondurensi. Floresta e Ambiente, v.21, n.2, p.261-268, 2014.

BORTOLETTO JÚNIOR, G. Avaliação da qualidade da madeira de Pinus merkusii para produção de lâminas. Scientia Forestalis, v. 36, n. 78, p. 95-103, 2008.

EISFELD, R. L., NASCIMENTO, F. A. F. Mapeamento dos plantios florestais do estado do Paraná: Pinus e Eucalyptus. Curitiba: Instituto de Florestas do Paraná, 2015. 76p. 
IAPAR - Instituto Agronômico do Paraná. Secretaria da Agricultura e do Abastecimento, Londrina, PR. Monitoramento agroclimático do Paraná. Cartas Climáticas do Paraná. $2015 \mathrm{a}$.

IAPAR - Instituto Agronômico do Paraná. Secretaria da Agricultura e do Abastecimento, Londrina, PR. Monitoramento agroclimático do Paraná. Médias Históricas das Estações do IAPAR. 2015b.

JUIZO, C. G. F., LOIOLA, P. L., ZEN, L. R., MARCHESAN, R., CARVALHO, D. E., BILA, N. F., ... \& KLITZKE, R. J. Variação radial das propriedades físicas da madeira de Pinus patula plantados em Moçambique. Pesquisa Florestal Brasileira, v. 35, n. 83, p. 285-292, 2015.

LIEBSCH, D.; OLIVEIRA, E. B.; MOREIRA, J. M. M. Á. P.; MIKICH, S. B. Inventário e aplicativo computacional para avaliação de danos causados por macaco-prego em plantios de pinus. Comunicado Técnico 384. EMBRAPA, 2016.

MATTOS, B. D.; GATTO, D. A.; STANGERLIN, D. M.; CALEGARI, L.; MELO, R. R.; SANTINI, E. J. Variação axial da densidade básica da madeira de três espécies de gimnospermas. Revista Brasileira de Ciências Agrárias, v.6, n.1, p. 121126, 2011.

ORWA, C.; MUTUA, A.; KINDT, R.; JAMNADASS, R.; SIMONS, A. Pinus patula a tree reference and selection guide. Agroforestry Database version 4.0, 2009.

REZENDE, M. A., COSTA, V. E., ARONI, A. S., \& SEVERO, E. T. D. Estudo das variações de densidade da madeira do $L i$ quidambar styraciflua e do Pinus tecunumannii, como parâmetros de qualidade para a produção de móveis. Holos Environment, v. 7, n. 1, p. 60-71, 2017.

RIBASKI, N. G., HILLIG, É., MIRANDA, G. M., CATAPAN, D. C. Análise da Produção de Compensados do Estado do Paraná. Braz. J. of Develop., v. 3, n. 1, p. 2-33, 2017.

TREVISAN, R.; HASELEIN, C. R.; MELO, R. R.; STANGERLIN, D. M.; BELTRAME, R.; GATTO, D. A.; CALEGARI, L. Variação radial da densidade básica da madeira de Eucalyptus grandis W. Hill exMaiden. Floresta, v.38, n.3, p. 553-559, 2008.

TRIANOSKI, R., MATOS, J. L. M. D., IWAKIRI, S., \& PRATA, J. G. Avaliação das propriedades mecânicas da madeira de espécies de Pinus tropicais. Scientia Forestalis, Piracicaba, v. 42, n. 101, p. 21-28, 2014.
VALÉRIO, A. F.; WATZLAWICK, L. F.; SANTOS, R. T.; SILVESTRE, R.; KOEHLER, H. S. Determinação da densidade básica da madeira de peroba (Aspidosperma polineuron Muell. Arg.) ao longo do fuste. Caatinga, v.21, n.3, p 54-58, 2008.

VIEIRA, M. C., BRITO, E. O., GONÇALVES, F, G. Evolução Econômica do Painel Compensado no Brasil e no Mundo. Floresta e Ambiente, v. 19, n.3, p.277-285, 2012. 